\title{
Dynamic Stabilization of an Automotive using Pattern Recognition
}

\author{
Sudeep.V \\ SITE, VIT University. \\ Vellore, Tamil Nadu, India
}

\author{
Pradeep Freddy. A \\ SITE, VIT University. \\ Vellore, Tamil Nadu, India
}

\author{
Avinash Choudhary. A.R \\ SITE, VIT University \\ Vellore, Tamil Nadu, India
}

\begin{abstract}
To err is human! Preventing errors using inhuman devices can save disasters. In this article we propose an inhuman robotic system that can prevent distance related disasters or accidents. The system running on the proposed algorithm calibrates itself with the subject which is identified during the initial stages and maintains a constant distance with the subject preventing clashing of the system with the subject. The system can be used in varieties of applications which are discussed briefly in this paper.
\end{abstract}

\section{Keywords}

Image Threshold, Shape Analysis, Pattern recognition, color recognition

\section{INTRODUCTION}

Image processing using robots mounted with camera's or sensors is a method of converting an image into digital form and perform some operations on it, in order to extract useful information from it. It is a type of stimulus or signal dispensation in which input is given as an image or a video stream or a photograph and the output is the characteristic response associated with that image or video frame. Image processing basically included the following three steps

- Importing the image with camera's or sensors.

- Analyzing the image which includes spotting patterns.

- Output is the last stage which includes the response as defined to the changes in the image.

Modern image capturing devices like digital camera, sensors, video recorders capture big amount of data. Here the data captured gives the same meaning as the data present in the environment. Main applications are in autonomous navigation, finding of abnormality in medical images or the predicting volcanic eruption. Simple task such as human face detection which are already solved, are commercially available in digital cameras. However for complex tasks to produce semantically appropriate interpretations and enhance recognition results, system must incorporate knowledge to be used efficiently. The big amount of data that is processed requires sophisticated representations that are both efficient and robust with respect to noise and distortions in the measure.

\section{LITERATURE SURVEY}

\subsection{Pattern Recognition}

For a robot to sense the picture, it must be able to find patterns, and recognize these patterns as objects also as per the type of learning procedure used to generate the output value[7,8,9], Pattern recognition is generally categorized into

- Supervised learning

- Unsupervised learning

- $\quad$ Semi supervised learning
Supervised learning assumes a set of training data, consisting of a set of instances that have been properly hand labeled with the correct output. The learning procedure then generates a model that attempts to meet two conflicting objectives: "Perform as well as possible" on the training data.On the other hand, Unsupervised learning, assumes training data that has not been hand labeled, and attempts to find inherent patterns in the current data that can then be used to determine the correct output value for new data instances. A combination of the above two has recently been explored is semi-supervised learning, which uses a combination of labeled and unlabeled data. Here we introduce new method for detecting and locating objects of interest within a colour scene under very strong variability's in lighting conditions, object shape and pigmentation. This method is based on Nearest neighbor classification and Multiunit-Condensing techniques and is applied to implement the vision subsystem of a robotic citric harvesting device [2,].

Recent advances in computer vision have given rise to a robust and invariant visual pattern recognition technology that is based on extracting a set of characteristic features from an image. Such features are obtained with the Scale Invariant Feature Transform (SIFT) [7], that represents the variations in brightness of the image around the point of interest. Recognition performed with this type of features has been shown to be quite robust in realistic settings. This paper describes the application of this particular visual pattern recognition technology to a variety of robotics applications: object recognition, navigation, manipulation, human-machine interaction. The following sections describe the technology in more detail and present a business case for visual pattern recognition in the field of robotics and automation $[5,6]$.

\subsection{Image Threshold}

This is the simplest form of image fragmentation or segmentation. It is one of the oldest problems in automated image analysis. Basically there are two types of threshold:

- global threshold

- locally adaptive threshold.

Often Global threshold does not work satisfactorily due to the variability in the gray level intensities and because of noise. The working of locally adaptive threshold method is as follows.

1. They build a threshold surface that is a function on the image domain.

2. Threshold the image with this threshold surface.

\subsection{Threshold Categories[4]}

We categorize the threshold methods in six groups according to the information they exploiting. These categories are: 
1. Histogram shape-based methods, where, for example, the peaks, valleys and curvatures of the smoothed histogram are analyzed [1].

2. Clustering-based methods, where the gray-level samples are clustered in two parts as background and foreground object or alternately are modeled as a mixture of two Gaussians [1].

3. Entropy-based methods result in algorithms that use the entropy of the foreground and background regions, the cross-entropy between the original and binaries image, etc[1].

4. Object attribute-based methods search a measure of similarity between the gray-level and the binaries images, such as fuzzy shape similarity, edge coincidence, etc[1].

5. The spatial methods use higher-order probability distribution and/or correlation between pixels [1].

6. Local methods adapt the threshold value on each pixel to the local image characteristics [1].

\subsection{Shape Analysis and Color Recognition}

Shape analysis deals with automatic analysis of geometric shape. All objects obtained by modeling, or by extracting shape from 2D or 3D images, has to be simplified before a comparison can be made. The simplified representation of the objects is often called as shape descriptor. Most of the important information are carried out by these simplified representations, which is easier to handle, to store and to compare the shapes directly. Strictly speaking a complete shape descriptor is a representation that is used to completely reconstruct the original object[2].

Color recognition is the ability of an organism or machine to distinguish objects based on the wavelengths (or frequencies) of the light they emit, transmit,or reflect. Colors can be measured and quantified in numerous ways. Human's perception of colors is a subjective process whereby the brainresponds to the stimuli that are produced when incoming light reacts with the several types of cone photorecetors in the eye.

Visual recognition of patterns is a basic capability of most advanced species in nature. A big percentage of the human brain is devoted to visual processing with a substantial portion of this area used for pattern recognition (see references [3] for an in-depth treatment of the subject). Visual pattern recognition enables a variety of tasks such as object and target recognition, navigation, grasping and manipulation, among others. Advances in camera technology have dramatically reduced the cost of cameras making them the sensor of choice for robotics and automation. Vision provides a variety of cues about the environment (motion, color, shape, etc.) with a single sensor. Visual pattern recognition solves some fundamental problems in computer vision: correspondence, pose estimation, and structure from motion. Therefore, visual pattern recognition is considered to be an important, costefficient primitive for robotics and automation systems.

\section{PROPOSED ALGORITHM}

Algorithm for stabilization using pattern recognition:

\subsection{Algorithm DSAP (Video Stream)}

//Input: a live video stream from the camera/sensors mounted on the robot

//Output: instruction which adjust the position of the robot

Start

IArea<- 0

Input live stream of frames from the camera or sensors

Initialize color and shape of the subject to be recognized

Identify and refine the object of Interest from the subject by threshold so as to extract only the object of interest i.e the threshold pattern from the subject

Draw contour around the object of Interest from the subject

Calculate the area of the shape within the contour

Assign the calculated area to IArea variable

CallRunning(IArea)

Stop

\subsection{Algorithm Running(IArea)}

//Input: area of the object of interest from the subject

//Output: instruction to move the robot based on the conditions

Start

while area $!=$ IArea

if area $>$ IArea

move robot or automotive backward until

area $=$ IArea

end if

if area $<$ IArea

move robot or automotive forward until area $=$ IArea

end if

end while 


\section{RESULTS AND DISCUSSION}

\section{Expected Behavior:}

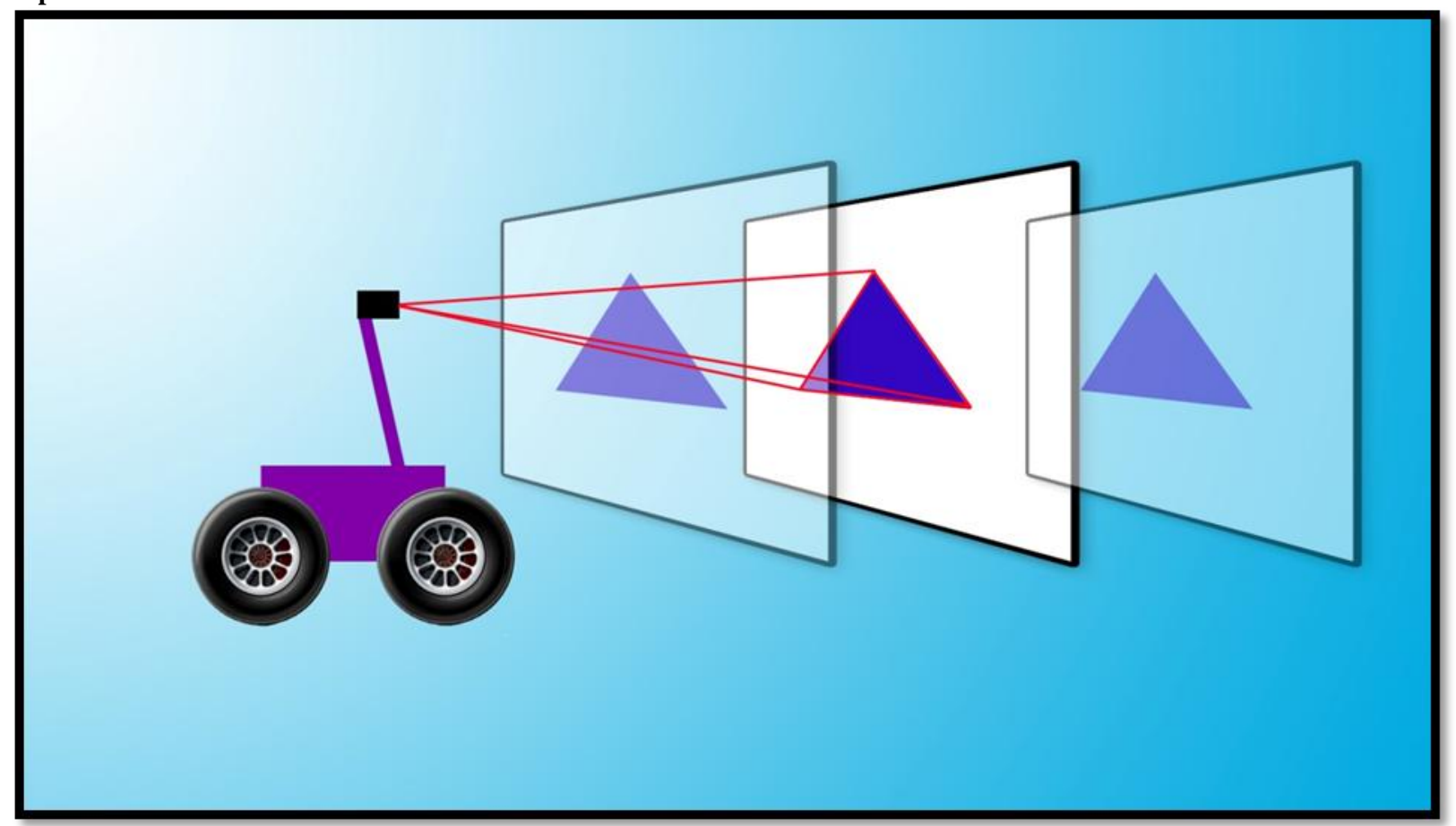

Fig 1

The diagram illustrated above and the algorithm in the previous segment clearly explains the working of the Robotic Automotive system.

The diagram depicts a robotic automotive system with a camera mounted on it to capture live stream of video for further processing. The white screen depicts the subject on which a blue triangle is the object of interest which is identified by threshold method. Threshold allows the system to identify the object of interest by filtering all the colors except the color of the object of interest. A contour is drawn around the object of interest (a triangle here) and the area of the object of interest is calculated and initialized. Having this area continuously the object of interest in the subject is checked, if the area increases or decreases it means that the subject has moved towards or away from the robotic automotive. Adjustments are made to the robotic automotive to take care that the area of the triangle remains the same, in other words the distance between the robotic automotive and the subject remains same.

\section{CONCLUSION AND FUTURE WORK}

In this paper, we proposed a system to identify patterns in a frame by the method of image threshold, shape and color recognition and image processing to extract information from the live stream of frames. An ASAP algorithm is proposed to do the above mentioned tasks. From the experimental results it is shown that this technique is capable to identify patterns in an image and take necessary action to adjust the automotive.

The following picture illustrates about the future enhancements:
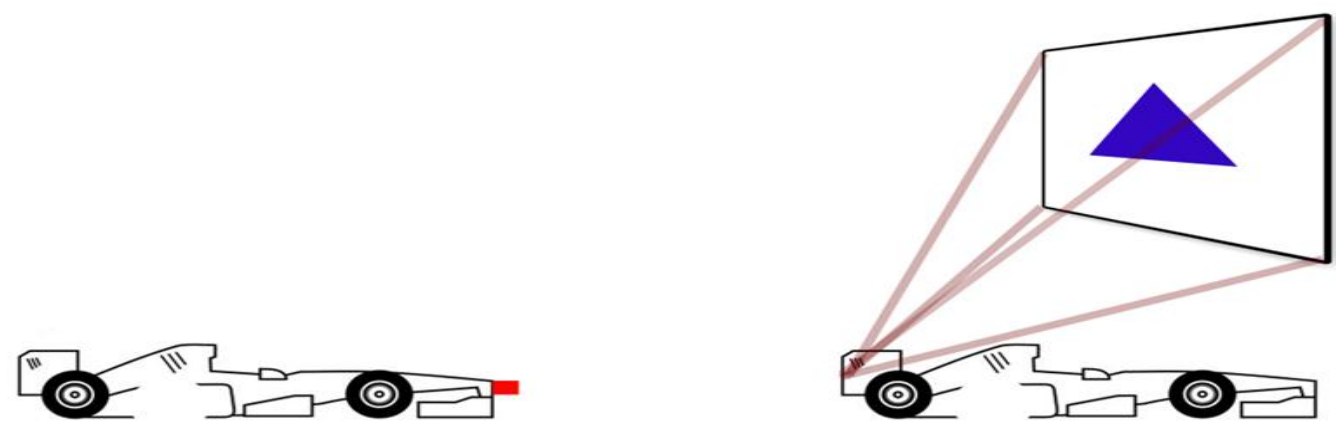


\section{REFERENCES}

[1] Mehmet Sezgin and $\mathrm{Bu}$ "lent Sankur, "Survey over image thresholding techniques and quantitative performance evaluation", Journal of electronic imaging, Vol. 13(1), pp. 146-165 January 2004.

[2] Francisco Ferri and Enrique Vidal , "Colour image segmentation and labeling through multiedit-condensing Pattern Recognition Letters" Journal of pattern recognition society, Vol. 13(8), pp. 561-568, August 1992.

[3] D. J. Felleman and D. C. Van Essen, "Distributed hierarchical processing in the primate cerebral cortex," Cerebral Cortex, Vol. 1(1), pp. 1-47, 1991.

[4] M. Sezgin and B. Sankur, "Comparison of thresholding methods for non-destructive testing applications,' IEEE ICIP'2001, Intl. Conf. Image Process., pp. 764-767, 2001.
[5] O. Carmichael and M. Hebert, "Shape-based recognition of wiry objects" in CVPR, pp. 401-408, 2003.

[6] S. Belongie, J. Malik and J. Puzicha, "Shape matching and object recognition using shape contexts". PAMI, 24(4): pp. 509-522, 2002.

[7] S. K. Nayar, and R. M. Bolle, "Reflectance Based Object Recognition", International Journal of Computer Vision, Vol. 17, No. 3, pp. 219- 240, 1996.

[8] Th. Gevers and Arnold W.M. Smeulders, "Color Based Object Recognition”, Pattern Recognition, 32, pp. 453 464, March 1999.

[9] D. Lowe, "Local feature view clustering for 3d object recognition," in Proc. of the 2001 IEEE Conf. on Computer Vision and Pattern Recognition, 2001. 